\title{
A rare life-threatening disease: unilateral kidney compressed by huge chronic spontaneous retroperitoneal hemorrhage
}

This article was published in the following Dove Press journal:

Therapeutics and Clinical Risk Management

\author{
Hao-Yuan Lu',* \\ Wei Wei ${ }^{2, *}$ \\ Qi-Wei Chen',* \\ Qing-Gui Meng' \\ Gao-Hua Hu' \\ Xian-Lin $\mathrm{Yi}^{1,3}$ \\ Xian-Zhong Bai' \\ 'Department of Urology, Tumor \\ Hospital of Guangxi Medical \\ University and Guangxi Cancer \\ Research Institute, Nanning \\ 53002I, China; ${ }^{2}$ Department of \\ Radiology, Tumor Hospital of \\ Guangxi Medical University and \\ Guangxi Cancer Research Institute, \\ Nanning 53002 I, China; ${ }^{3}$ Hubei \\ Engineering Laboratory for Synthetic \\ Microbiology, Wuhan Institute of \\ Biotechnology, Wuhan 430075, China \\ *These authors contributed equally \\ to this work
}

\begin{abstract}
Objectives: To study an uncommon life-threatening disease, spontaneous retroperitoneal and perirenal hemorrhage.

Case descriptions: A 69-year-old male presented with pain in the left waist and back of 1 month duration. The renal abscess was suspected by magnetic resonance imaging before operation. The perirenal hematoma was cleaned by operation. In another case, the patient had a functional solitary left kidney compressed by a huge retroperitoneal mass and uropenia appeared.

Results: The first patient died of adult respiratory distress syndrome after surgery. The second patient died of cardiac insufficiency and pulmonary embolism on the second day after evacuation of retroperitoneal hematoma.

Conclusion: Conservative surgery, such as selective arterial embolization, is a reasonable approach in patients with chronic spontaneous retroperitoneal and perirenal space hemorrhage and with poor general condition. We strongly recommend drainage or interventional therapy, but not a major surgery, in patients with poor condition.
\end{abstract}

Keywords: kidney, spontaneous, retroperitoneal, hemorrhage, surgery

\section{Plain language summary}

Unilateral kidney compressed by huge chronic spontaneous retroperitoneal hemorrhage is a rare life-threatening disease.

Conservative surgery is a reasonable approach in patients with chronic spontaneous retroperitoneal and perirenal space hemorrhage and with poor general conditions.

Drainage or interventional therapy may be a reasonable choice, but not a major surgery, in a patient with poor conditions.

\section{Introduction}

Spontaneous retroperitoneal hemorrhage is an uncommon life-threatening disease. ${ }^{1}$ The possible causes are anticoagulant therapy, isolated coagulation abnormalities, neoplasm, simple renal cyst, renal vascular diseases, and rupture of kidney. ${ }^{2-5}$

Diagnosis of spontaneous retroperitoneal hemorrhage is often delayed because the manifestation of its signs is late in the clinical course. ${ }^{5}$

The survival of patients is dependent on rapid, accurate diagnosis and appropriate management. Delayed diagnosis and treatment often means increased morbidity and mortality. Surgery, considered as the gold standard previously, shows absolute efficacy as definitive treatment and offers a favorable prognosis. However, it still lacks level I evidence for the best treatment. ${ }^{5,6}$
Correspondence: Xian-Lin Yi of Guangxi Medical University and Guangxi Cancer Research Institute, Nanning 53002I, People's Republic of China

Tel +86 | 529652 |23।

Email yzztx@।26.com 

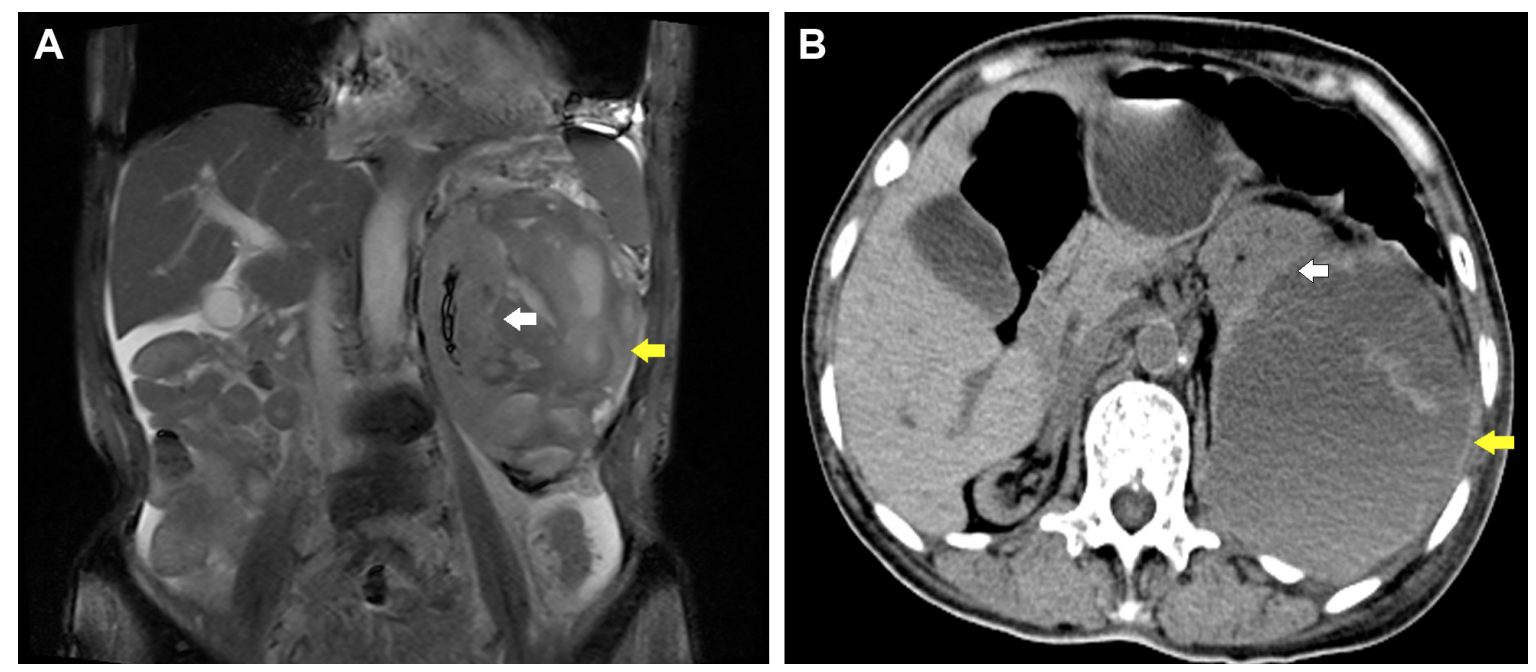

Figure I Imaging for Cases I and 2.

Notes: (A) Magnetic resonance imaging of the abdomen on presentation of Case I. Multiple cystic lesions were seen with short TI and long or short T2 signals (white arrow); left kidney was pushed and compressed by the mass (yellow arrow). (B) Computed tomography scan shows a huge mass from the left side of retroperitoneal to iliac fossa (yellow arrow) in Case 2; the functional solitary left kidney was pushed and compressed by the mass (white arrow).

Here, we report two patients who presented to the urologist with unilateral kidney compressed by huge chronic spontaneous retroperitoneal hemorrhage. The two cases died of multiple organ dysfunction syndrome not long after open exploratory surgery.

\section{Case I}

In December, 2016, a 69-year-old male presented with pain in the left waist and back of 1 month duration, he thought it was because of the cold wind. He also had accompanying symptoms of chest tightness, dyspnea, and cough. A chest computed tomographic (CT) scan showed bronchitis, and the waist and back pain aggravated again after anti-inflammatory treatment. A chest X-ray showed pleural effusion with atelectasis of the left lower lobe. Pulmonary ventilation showed mild restrictive pulmonary dysfunction, decrease in lung diffusion function, and normal range of airway resistance. A CT scan and magnetic resonance imaging (MRI) of the abdomen was obtained (Figure 1A). Laboratory test showed coagulation abnormality (Table 1). Although the patient has dyspnea before the operation, he did not have adults respiratory distress syndrome.

$\mathrm{CT}$ and MRI images revealed spontaneous perirenal hemorrhage of the left kidney. The diagnosis was confirmed by operation and pathology (Figure 2), but the origin of the mass was unclear simply based on imaging.

Abnormal MRI showed an $11.7 \times 10.0 \mathrm{~cm}$ mass around the left renal parenchyma, constricting it. T1-weighted image (T1WI) sequence showed high signal, and T2-weighted image (T2WI) sequence showed mixed signal and no enhancement.
The signal of left kidney was uneven with a blurred outline. Multiple patchy and nonenhanced low-signal lesions compressed the left kidney. Multiple cystic lesions were seen with the short $\mathrm{T} 1$ and long or short T2 signals (Figure 1A). A large number

Table I Laboratory test results before surgery

\begin{tabular}{|c|c|c|c|}
\hline Test & Case I & Case 2 & $\begin{array}{l}\text { Normal } \\
\text { reference } \\
\text { range }\end{array}$ \\
\hline \multicolumn{4}{|l|}{ Blood } \\
\hline PT, s & 15 & 45.9 & $9-13$ \\
\hline PT INR & 1.26 & 3.73 & $0.8-1.2$ \\
\hline Activated partial & 60.1 & 76.2 & $20-40$ \\
\hline \multicolumn{4}{|l|}{ thromboplastin time, $s$} \\
\hline $\mathrm{TT}, \mathrm{s}$ & 16.5 & 15.6 & $|4-2|$ \\
\hline $\mathrm{FIB}, \mathrm{g} / \mathrm{L}$ & 2.72 & 4.25 & $2-4$ \\
\hline D-dimer, mg/L & 12.19 & 2.15 & \\
\hline Platelets, /L & $386 \times 10^{9}$ & $390 \times 10^{9}$ & $100-300 \times 10^{9}$ \\
\hline Hemoglobin, g/L & 87.0 & 54.0 & $13 \mid-172$ \\
\hline $\mathrm{MCV}, \mathrm{fL}$ & 86.0 & 85.7 & $86-100$ \\
\hline $\mathrm{MCH}, \mathrm{pg}$ & 28.4 & 27.2 & $26-31$ \\
\hline White blood cell count, /L & $21.47 \times 10^{9}$ & $20.86 \times 10^{9}$ & $3.97-9.15 \times 10^{9}$ \\
\hline Neutrophils, \% & 88.3 & 92.6 & $45-77$ \\
\hline Lymphocytes, \% & 5.5 & 2.5 & $20-40$ \\
\hline CRP, mg/L & 79.69 & 45.41 & $0-10$ \\
\hline hs-CRP, mg/L & 4.83 & 5.62 & $0-3$ \\
\hline IgG, g/L & 11.53 & 8 & $8-16$ \\
\hline Alexin C3, g/L & 0.63 & 0.6 & $0.9-1.5$ \\
\hline Alexin C4, g/L & 0.12 & 0.19 & $0.2-0.4$ \\
\hline Creatinine, $\mu \mathrm{mol} / \mathrm{L}$ & 548 & 246 & $53-123$ \\
\hline Total protein, g/L & 51.8 & 62.6 & $60-80$ \\
\hline Globulin, g/L & 20.9 & 32.9 & $25-35$ \\
\hline Albumin:globulin ratio & 0.68 & I.II & $\mathrm{I}-2.5$ \\
\hline \multicolumn{4}{|l|}{ Urine } \\
\hline Blood, $\mu \mathrm{L}$ & 5 & 0 & $0-5$ \\
\hline
\end{tabular}

Abbreviations: CRP, C-reactive protein; FIB, fibrinogen; IgG, immunoglobulin G; INR, international normalized ratio; $\mathrm{MCH}$, mean corpuscular hemoglobin; MCV, mean corpuscular volume; PT, prothrombin time; TT, thrombin time. 


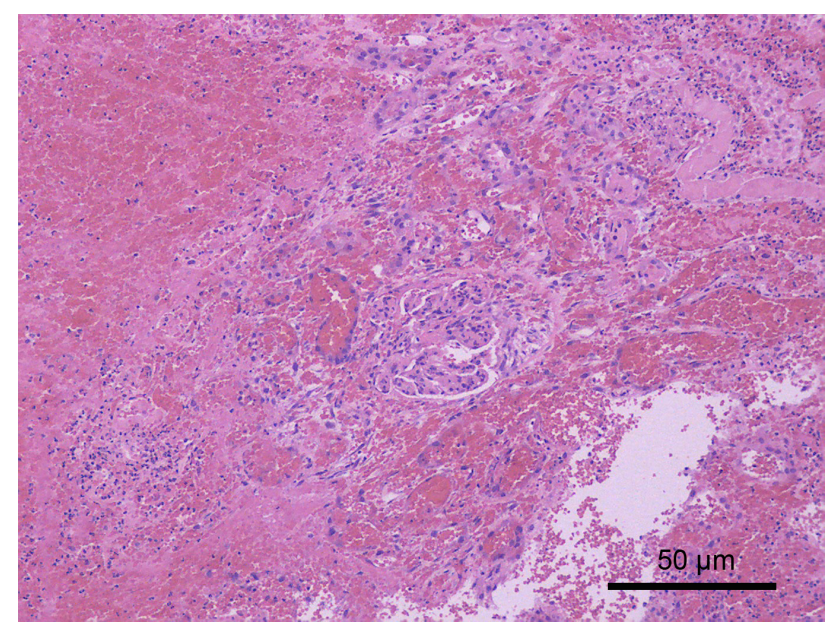

Figure 2 Pathologic findings of Case I.

Notes: The majority of mass consisted of blood clots and fibrinous exudate cells, and a few broken and necrotic glomerular and renal tubules were seen. No evidence of malignancy and no bacteria were found in the necrotic foci. PAS and D-PAS were negative. Magnification $\times 100$

Abbreviations: PAS, periodic acid-Schiff; D-PAS, D-periodic acid-Schiff stain.

of blood clots around the left kidney were seen in exploratory laparotomy. Hemorrhage and necrosis were discovered in the pseudocapsule. Lymph nodes of renal hilar were not enlarged. The perirenal hematoma was cleaned by pathology. The patient died of adult respiratory distress syndrome after the operation.

\section{Case 2}

In December 2016, a 67-year-old female presented with left leg swelling of 13 days duration because of sprain of left ankle. She had a history of polycythemia vera disease for 20 years and hypertension for 5 years. Anticoagulation with warfarin and low-molecular-weight heparin therapy was received by the patient for lower extremity vein thrombosis. A CT scan showed a huge mass from the left side of retroperitoneal to iliac fossa (Figure 1B). The left ventricular systolic function was normal on color Doppler echocardiography. The functional solitary left kidney was pushed and compressed by the mass and uropenia appeared soon after admission. Hemoglobin declined from 77 to $50 \mathrm{~g} / \mathrm{L}$. Creatinine increased from 122 to $246 \mu \mathrm{mol} / \mathrm{L}$ (Table 1).

A vena cava filter was implanted, anticoagulation treatment was stopped, and fresh frozen plasma was infused in the perioperative period. A left ureteral stent implantation, an exploratory laparotomy, and evacuation of retroperitoneal hematoma were performed because of hemodynamic instability.

A huge hematoma was seen in the retroperitoneum. Renal fascia and fat capsule were intact. The left kidney was normal, with no bleeding; no tumor and no hematoma. No pre-existing anatomic lesion was seen. The patient died of cardiac insufficiency and pulmonary embolism on the second day after the operation.

\section{Ethics approval}

The study was approved by the Ethics Committee of Tumor Hospital of Guangxi Medical University. The next of kin of both patients provided written informed consent for the case details and accompanying images to be published.

\section{Discussion}

Spontaneous retroperitoneal hemorrhage is a rare disease with variable etiology, which usually occurs in patients receiving anticoagulant therapy. ${ }^{5}$ The incidence of spontaneous retroperitoneal hemorrhage in patients receiving oral anticoagulants is $0.1 \%-0.6 \%{ }^{4,6}$ Coagulation abnormalities were present in both the patients discussed here and anticoagulation was received by Case 2 (Table 1).

Diagnosis of spontaneous retroperitoneal hemorrhage is often delayed. CT is thought to be the best imaging modality, but does not have enough sensitivity in identifying an underlying neoplasm., ${ }^{2,4}$ MRI is also useful.

Low signal on T2WI with low to intermediate signal on T1WI usually demonstrates acute perirenal hemorrhage. ${ }^{5}$ However, high signal on T1WI and T2WI, low signal on T1WI, and high signal on T2WI usually demonstrates chronic perirenal hemorrhage.

It is confirmed that there was a mixture of a fresh and a chronic organized hematoma, so that a renal abscess was considered by MRI before the operation in case 1 . The subacute clinical course may also permit the person to obtain an inaccurate diagnosis on $\mathrm{CT} .{ }^{6}$

Possible treatments include conservative measures, selective arterial embolization, evacuation, and nephrectomy. Nephrectomy was previously first-line treatment, but it is only carried out if malignancy is considered. Rebleeding is one of the risks of immediate surgical removal. ${ }^{7}$ Acute spontaneous perinephric hematoma must be treated early, and delaying the treatment can be life-threatening. ${ }^{2}$

Selective arterial embolization is safe and reliable, with stable long-term renal function for patients with acute hemorrhage ${ }^{3,6,8}$ Mao et al reported the hemorrhage involving pararenal space as Type III, which may involve subcapsular and perirenal spaces. ${ }^{3}$

Conservative surgery is a reasonable approach in patients with chronic and Type III spontaneous perirenal hemorrhage and with poor general conditions.

Although urination was significantly increased after the operation when the pressure on kidney was relieved, oliguria presented soon after in the cases reported here. We strongly recommend drainage of hematoma, guided by ultrasound or $\mathrm{CT}$, but not major surgery, especially in a patients with poor condition. ${ }^{6,8}$ If abscess is suspected, puncture is necessary 
before exploration. Otherwise, a watch-and-wait approach may be a safe and less-invasive option. ${ }^{7}$ In Case 2, the hemoglobin level decreased from 77 to $50 \mathrm{~g} / \mathrm{L}$, which indicated persisting hemorrhage; renal arterial digital subtraction angiography or computed tomography angiography (CTA) would be useful to find the causes and perform interventional therapy. ${ }^{6}$

It is reported that spontaneous retroperitoneal hemorrhage may result from abdominal vascular rupture, rheumatologic disease, renal tumors, lumbar artery or venous rupture in anticoagulant administered and hemodialysis patients. . $^{5,6,9,10}$ We must be wary of spontaneous retroperitoneal hemorrhage if these patients present with flank pain, anemia, and hemodynamic instability.

The major indications of operation include hemodynamic instability, abdominal compartment syndromes, and impaired respiratory and cardiovascular functions, but the latter two often mean treatment failure. ${ }^{10}$ Therefore, once the diagnosis has been confirmed, intervention must be started because early diagnosis and appropriate treatment can decrease the mortality and morbidity. ${ }^{9}$

It may be challenging to manage the coexisting embolic disease. Anticoagulation must be withheld, but can be restarted after 3 days in conservative therapy as reported. ${ }^{11}$

\section{Data sharing statement}

Additional unpublished data are available by request to the lead author.

\section{Acknowledgments}

This work was supported by Hubei postdoctoral innovation post-fund, Wuhan postdoctoral innovation post-fund, the self-financing research of the Health Department of Guangxi Autonomous Region (Z2016494, Z2016499), and the National Natural Science fund (81560428). HL, WW and QC are joint first authors.

\section{Author contributions}

XLY, QGM, HYL, GHH, XZB: study design. XLY, HYL, WW, QWC: acquisition, analysis, and interpretation of data, drafting the manuscript. All authors have read and approved the final manuscript. All authors contributed toward data analysis, drafting and critically revising the paper and agree to be accountable for all aspects of the work.

\section{Disclosure}

The authors report no conflicts of interest in this work.

\section{References}

1. Daskalopoulos G, Karyotis I, Heretis I, Anezinis P, Mavromanolakis E, Delakas D. Spontaneous perirenal hemorrhage: a 10-year experience at our institution. Int Urol Nephrol. 2003;36(1):15-19.

2. Mckinnon K, Baker K, Ravi R. Case of spontaneous bilateral perinephric hematomas. Int J Urol. 2014;22(1):137-138.

3. Mao Y, De Oliveira IS, Hedgire S, Prapruttam D, Harisinghani M. Aetiology, imaging features, and evolution of spontaneous perirenal haemorrhage. Clin Radiol. 2017;72(2):175.

4. Ibrahim W, Mohamed A, Sheikh M, et al. Antiplatelet therapy and spontaneous retroperitoneal hematoma: a Case Report and Literature Review. Am J Case Rep. 2017;18:85-89.

5. Tseng J, Leshen M, Chapman T, Scott R, Kalinkin O. Diagnosis and management of spontaneous lumbar venous retroperitoneal hematoma in setting of deep venous thrombosis: a case report and algorithm. Case Rep Radiol. 2016;2016:3183985.

6. Chan YC, Morales JP, Reidy JF, Taylor PR. Management of spontaneous and iatrogenic retroperitoneal haemorrhage: conservative management, endovascular intervention or open surgery? Int J Clin Pract. 2008;62(10):1604-1613.

7. Diaz JR, Agriantonis DJ, Aguila J, Calleros JE, Ayyappan AP. Spontaneous perirenal hemorrhage: what radiologists need to know. Emerg Radiol. 2011;18(4):329-334

8. Vaidyanathan S, Soni BM, Hughes PL, et al. How should an infected perinephric haematoma be drained in a tetraplegic patient with baclofen pump implanted in the abdominal wall? - A case report. BMC Urol. 2002;2:9.

9. Hwang NK, Rhee H, Kim IY, et al. Three cases of spontaneous lumbar artery rupture in hemodialysis patients. Hemodial Int. 2017;21(1): E18-E21.

10. Maruyama T, Abe M, Furukawa T, et al. Retroperitoneal hematoma in a patient with advanced chronic kidney disease receiving warfarin therapy. Intern Med. 2016;55(9):1153-1158.

11. White DJ, Lytle FT. Femoral neuropathy following spontaneous retroperitoneal hemorrhage after cardiac surgery: a case report. AA Case Rep. 2017;8(8):203-205.
Therapeutics and Clinical Risk Management

\section{Publish your work in this journal}

Therapeutics and Clinical Risk Management is an international, peerreviewed journal of clinical therapeutics and risk management, focusing on concise rapid reporting of clinical studies in all therapeutic areas, outcomes, safety, and programs for the effective, safe, and sustained use of medicines. This journal is indexed on PubMed Central, CAS,

\section{Dovepress}

EMBase, Scopus and the Elsevier Bibliographic databases. The manuscript management system is completely online and includes a very quick and fair peer-review system, which is all easy to use. Visit $\mathrm{http}: / / \mathrm{www}$.dovepress.com/testimonials.php to read real quotes from published authors. 